MODELING, IDENTIFICATION AND CONTROL, 1991, VOL. 12, NO. 1, 13-25

doi:10.4173/mic.1991.1.2

\title{
Using momentum conservation to control kinematically redundant manipulators $\dagger$
}

\author{
JAN RICHARD SAGLI $\ddagger$ and OLAV EGELAND
}

Keywords: Robotics, momentum conservation, redundant manipulators.

\begin{abstract}
A control scheme for the coordination of motion in a macro-micro manipulator system is presented. The motion of the end-effector is decoupled from the rest of the system using resolved acceleration control, while a slow, gross positioning is used for the macro part. To avoid saturated inputs and excessive use of energy for systems with limited fuel, the end effector is not decoupled from macro part motion, but from selected directions of the linear momentum of the total system. For the vehiclemanipulator case, the end-effector is decoupled also from the angular momentum. This leads to lower force and torque peaks in the actuators, and control force is used on the macro part only when it is necessary to reposition this to keep the micro part inside its workspace.
\end{abstract}

\section{Introduction}

In a macro-micro manipulator system the coordination of motion between the macro and micro part is important for obtaining improved performance. Utilizing the redundancy may among other things lead to better dynamical coordination, increased acceleration capabilities and smaller tracking errors. For a system with limited amounts of fuel, e.g. a spacecraft-manipulator system, low fuel requirements is of great interest. However, these advantages are only obtained if a good control strategy is applied.

The satellite-manipulator system can be treated as a macro-micro manipulator system as described by Egeland (1987), Khatib (1986) or Salisbury and Abramowitz (1985) where the manipulator gives a fast and accurate end-effector motion, and the spacecraft is a slower positioning part which provides a large workspace.

A macro-micro manipulator is a redundant manipulator which has an internal or nullspace motion. For the satellite-manipulator system it is natural to describe the internal motion in terms of position and orientation of the spacecraft. However, internal motion can also be described in terms of linear and angular momentum of the total system; which is useful in the development of the controller. Decoupling of endeffector and internal motion for macro-micro manipulators can be achieved using an augmented Jacobian in the resolved acceleration control scheme (Egeland (1987)).

Received 7 January 1991.

† This project is supported by the Norwegian Space Center.

$\ddagger$ Center for Robotics Research, Division of Engineering Cybernetics, The Norwegian Institute of Technology, 7034 Trondheim, Norway.

This paper was presented at the IEEE International Workship on Intelligent Motion Control, Istanbul, Turkey, 20-22 August 1990. It is reprinted from the Proceedings, pp. 541-546, with the permission of the IEEE. 
The computed torque algorithm gives a global linearization of the system, enabling the use of linear theory, with the drawback that information about the torque input constraints is lost in the transformation from torque space to acceleration space. Furthermore, it is well known that the computed torque algorithm gives reduced tracking accuracy with saturated inputs. Methods to cope with this problem are proposed by Dwyer, Fadali, Chen and Lee (1985) and Spong, Thorp and Kleinwaks (1986). When saturation occurs, these approaches both result in an increased use of nonsaturating joint actuators to reduce the tracking error.

The coordination of motion between spacecraft and manipulator is important for repair and servicing satellites with one or more manipulators. The manipulator inertia may be significant compared to the satellite inertia, which gives kinematic and dynamic coupling. It is possible to decouple satellite and manipulator motion using momentum compensation wheels or reaction jets, but this may require excessive use of control energy. Several researchers have addressed this problem.

Longman, Lindberg and Zedd (1987) proposed to use reaction wheels in the satellite to compensate for reaction torques at the manipulator base, and thereby decouple the rotation of the spacecraft from manipulator motion. The kinematics and dynamics of the manipulator were there modified to take into account the three translational degrees of freedom of the base. Vafa and Dubowsky $(1987 \mathrm{a}, \mathrm{b})$ introduced the concept of a virtual manipulator to simplify the kinematics and dynamics of spacecraft-manipulator systems. In their paper they used this technique to control endeffector position while keeping a constant satellite attitude using only the actuators in the manipulator. Nakamura and Mukherjee (1989) proposed a path planning scheme where both end-effector position and satellite attitude were controlled using manipulator actuators. Alexander and Cannon (1987) demonstrated that end-effector motion can be controlled without attitude control of the satellite when the thrust forces of the satellite are known.

In several applications it is interesting to control end-effector position while using the satellite for slow gross positioning. Normally, it is not necessary to decouple endeffector and satellite motion, but it can be useful to coordinate the motion of manipulator and satellite to keep the end-effector task inside the manipulators workspace. The energy consumption can then be considerably lower than if decoupling is used as in Longman et al. (1987), and the solution becomes more flexible than in Alexander and Cannon (1987), Nakamura and Mukherjee (1989) and Vafa and Dubowsky (1987a) where only manipulator torques are used.

As mentioned, it is also interesting to avoid saturated inputs leading to reduced tracking accuracy. However, the methods in Dwyer et al. (1985) and Spong et al. (1986) are best suited for feedback linearization for nonredundant manipulators, because the redundancy resolution will influence the end-effector tracking strongly if they are used for manipulators with redundant degrees of freedom. With these algorithms any trajectory given in joint space will be attempted tracked, and the redundancy will not be utilized to improve the end-effector task. However, this is not necessary since the important issue is to follow the end-effector trajectory, while internal motion should be used to obtain this. Also the redundancy resolution scheme using the task-priority strategy as in Nakamura, Hanafusa and Yoshikawa (1987) admits perfect tracking of the calculated path and get into trouble if actuators saturate.

In this paper we would like to focus on the possibilities for improved end-effector tracking characteristics obtained by a more efficient use of the actuators. A decoupling of end effector motion from macro part motion does not necessarily give the most 
efficient use of these. In many cases, a better choice is to decouple end-effector motion from the linear and/or angular momentum of the system. In this way, large peaks in the actuator torques are avoided by a more economical internal motion which leads to a distribution of the inputs in time.

In satellite manipulator control the same control scheme can be used, and in this case increased fuel efficiency is an additional effect. Here we focus on the decoupling of end-effector motion from satellite motion, and on the distribution of fast and slow motion to manipulator and satellite.

In this paper a control scheme is presented using the macro-micro manipulator approach. First we study a four link planar experimental manipulator to illustrate the possibilities in a simple system. We further extend this to a satellite-manipulator system which can be considered as a more general case. The satellite motion is controlled using wheels or reaction jets, but energy is saved as the end-effector motion in the dynamical controller is decoupled not from satellite motion but from the linear and angular momentum of the total system. A slow gross positioning is used for the satellite. The performance of the proposed controller is demonstrated in a simulation study using a model with 12 degrees of freedom.

\section{Modeling the macro-micro manipulator}

A macro-micro manipulator is a system which can utilize the normally heavy macro part for gross positioning purposes, while the micro part is used to perform the endeffector task. Thus it is natural to split up the $n$-dimensional vector $q$ of generalized coordinates in two subvectors defined by

$$
\boldsymbol{q}=\left(\begin{array}{c}
\boldsymbol{q}_{M} \\
\boldsymbol{q}_{\mu}
\end{array}\right)
$$

where the joints closest to the base belonging to the macro part are denoted $\boldsymbol{q}_{M}$ while the faster micro part joints are denoted $\boldsymbol{q}_{\mu}$. The equation of motion for the system is written

$$
\boldsymbol{M}(\boldsymbol{q}) \ddot{\boldsymbol{q}}+\boldsymbol{n}(\boldsymbol{q}, \dot{\boldsymbol{q}})=\boldsymbol{\tau}
$$

where $\boldsymbol{M ( q )}$ is the inertia matrix, $\boldsymbol{n}(\boldsymbol{q}, \dot{q})$ is the vector of Coriolis, centrifugal and friction terms, and the vector of generalized forces is

$$
\tau=\left(\begin{array}{c}
\tau_{M} \\
\tau_{\mu}
\end{array}\right) \text {. }
$$

where the subscripts are the same as for the generalized coordinates.

We want to control the manipulator in cartesian space, and choose to augment the task space with extra coordinates from internal motion as e.g. in Egeland (1988), such that a well defined square Jacobian can be written

$$
\dot{\boldsymbol{x}}=\mathrm{J}_{a}(q) \dot{q}
$$

which is the same as

$$
\left(\begin{array}{c}
\dot{x}_{e e} \\
\dot{x}_{M}
\end{array}\right)=\left(\begin{array}{ll}
J_{11} & J_{12} \\
J_{21} & J_{22}
\end{array}\right)\left(\begin{array}{c}
\dot{q}_{M} \\
\dot{q}_{\mu}
\end{array}\right)
$$

where $\boldsymbol{x}_{e e}$ are the end-effector coordinates and $\boldsymbol{x}_{M}$ are the augmented coordinates. 
Decoupling is then achieve as for macro-micro manipulators in Egeland (1987) using

$$
\tau=M(q) J_{a}^{-1}(q)\left[u_{a}-\dot{J}_{a} \dot{q}\right]+n(q, \dot{q})
$$

which is a modification of the resolved acceleration control scheme (Luh, Walker and Paul (1980 a)) for nonredundant manipulators. The new control vector is

$$
\boldsymbol{u}_{a}=\left(\begin{array}{l}
\boldsymbol{u}_{e e} \\
\boldsymbol{u}_{M}
\end{array}\right)
$$

and the system

$$
\ddot{\boldsymbol{x}}_{a}=\boldsymbol{u}_{a}
$$

is obtained.

It is assumed that the primary task is to track an end-effector reference, while the secondary task can be described by

$$
\boldsymbol{x}_{M}=\boldsymbol{q}_{M}
$$

and a reference can be given to this through a redundancy resolution. But, exact tracking of this reference is normally not of great importance, so one possibility is to use a filtered reference or lower bandwidth to control this task space direction to get a slow gross and less energy consuming positioning. Nevertheless, we propose that for the development of the dynamic controller with resolved acceleration control, it is advantageous to control selected directions from the linear and angular momentum instead of controlling satellite motion directly. Thus an alternative secondary task consists of a sufficient number of elements from the vector $\delta x_{c m}=\dot{x}_{c m} \delta t$ is defined by

$$
\dot{\boldsymbol{x}}_{c m}=\left(\begin{array}{c}
{ }^{I} \boldsymbol{P} \\
{ }^{I} \boldsymbol{H}
\end{array}\right) \text {, }
$$

where ${ }^{I} P$ and ${ }^{I} H$ are the linear and angular momentums. The $6 \times 12$ Jacobian matrix $\boldsymbol{J}_{c m}$ is defined by

$$
\dot{\boldsymbol{x}}_{\mathrm{cm}}=\boldsymbol{J}_{\mathrm{cm} \dot{\boldsymbol{q}}}
$$

and is calculated in a similar way as the end-effector Jacobian $\boldsymbol{J}_{e e}$ using eqns. (12) and (14). For the vehicle-manipulator case, the linear momentum is defined as

$$
{ }^{I} \boldsymbol{P}=\sum_{k=0}^{6} m_{k}{ }^{I} \dot{\boldsymbol{r}}_{G}
$$

where $I$ denotes inertial frame, 0 denotes vehicle frame, and frames $1,2, \ldots, 6$ denote manipulator frames. $r_{G}$ is the center of mass of the entire system defined as

$$
{ }^{I} r_{G}=\frac{\sum_{k=0}^{6} m_{k}{ }^{I} r_{k}}{\sum_{k=0}^{6} m_{k}} .
$$

The angular momentum around the center of mass is

$$
{ }^{I} \boldsymbol{H}=\sum_{k=0}^{6}\left({ }^{I} M_{k}{ }^{I} \omega_{k}+m_{k}{ }^{I} S_{k} \times{ }^{I} \dot{S}_{k}\right)
$$

where ${ }^{I} M_{k}={ }_{k}^{I} R^{k} M_{k I}{ }^{k} R$ is the inertia matrix of body $k$ around its center of mass in frame $I, \omega_{k}$ is the angular velocity of body $k$, and $s_{k}=r_{k}-r_{G}$ is the position of body $k$ relative to 
the centre of mass. ${ }^{k} M_{k}$ is the constant inertia matrix of body $k$ in frame $k$, and $R$ denotes a rotation matrix from the frame given by the subscript to the frame given by the superscript. The only external forces acting on the system are $\tau_{M}$, resulting in the following equation of motion for the total system:

$$
\left(\begin{array}{c}
{ }^{I} \dot{\boldsymbol{P}} \\
{ }^{I} \dot{\boldsymbol{H}}
\end{array}\right)=\tau_{M}
$$

The selection of momentum coordinates for use in the resolved acceleration control is highly dependent of the mechanical structure of the actual manipulator. The best result will be obtained if the directions are chosen where the momentums are most significant. Now, if the end-effector velocity $\dot{\boldsymbol{x}}_{e e}$ is specified and the macro part velocity $\dot{\boldsymbol{x}}_{M}$ is found by a redundancy resolution algorithm, it is possible to find a reference for ${ }^{I} P$ or ${ }^{I} H$ using (11) by first calculating the joint velocities $\dot{\boldsymbol{q}}_{\mu}$ from

$$
\dot{\boldsymbol{x}}_{e e}=J_{11} \dot{\boldsymbol{q}}_{M}+J_{12} \dot{q}_{\mu}
$$

When the secondary task is given as in eqn. (9) the solution

$$
\dot{\boldsymbol{q}}_{\mu}=\mathrm{J}_{12}^{-1}\left(\dot{\boldsymbol{x}}_{e e}-\mathrm{J}_{11} \dot{\boldsymbol{x}}_{M}\right)
$$

can be found using Gaussian elimination, provided that the matrix $J_{12}$ has full rank. This means that the same singularities as for the micro-manipulator have to be avoided.

\section{Coordination of macro- and micro part motion}

The macro and micro part must be coordinated so that

- The task is inside the manipulators workspace

- The end-effector tracking is accurate which requires that torque saturation should be avoided

- Excessive use of limited resources as control energy in general and reaction jet fuel in particular is avoided for a vehicle-manipulator system.

The workspace problem is solved through kinematics, while actuator efficiency is achieved through controller design. The main contribution of this paper is on the control part.

\section{Simulations on macro-micro manipulator}

\subsection{Planar experimental manipulator}

A simulation study is performed on the planar manipulator with four degrees of freedom shown in Fig. 1. The manipulator consists of a translational joint in the base and three rotational joints. The Denavit-Hartenberg parameters and the link masses are given in Table 1. The relationship to satellite control should be quite clear, observing that the first translational joint, the macro part, can be considered as a satellite moving in a linear space. In the simulations, a $5 \mathrm{~kg}$ load was put on the outmost link.

\subsection{Results}

The planar manipulator was considered as a macro-micro manipulator with the first heavy translational joint as the macro part. The primary task was to position and 


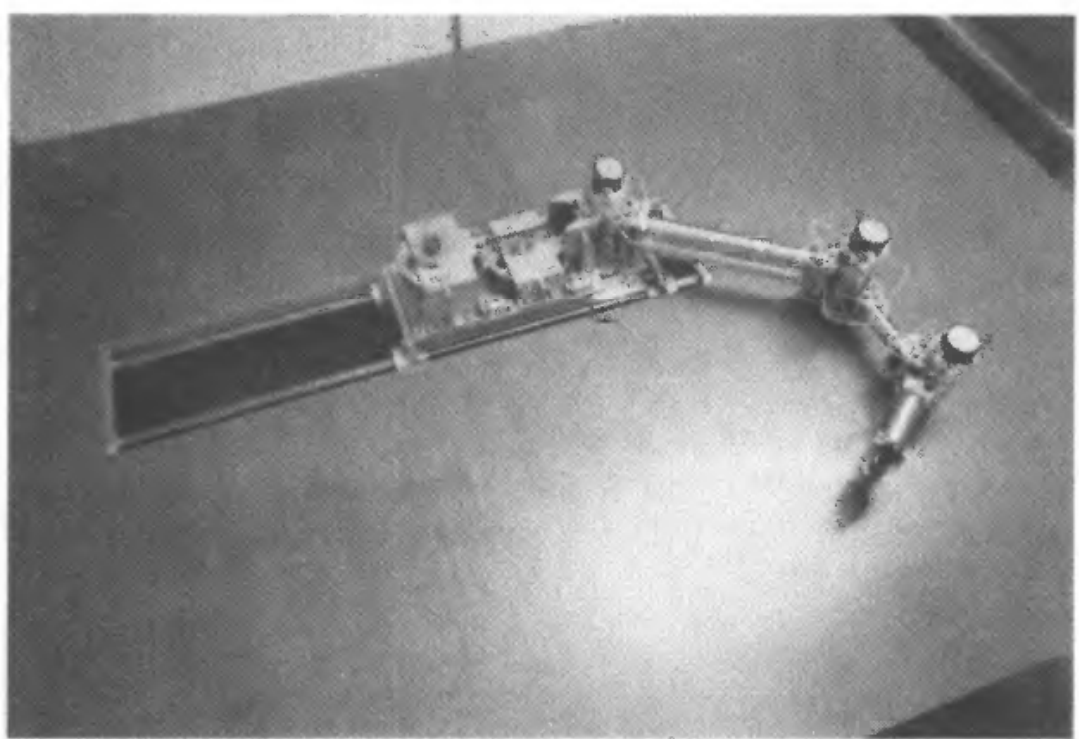

Figure 1. Four link planar manipulator.

\begin{tabular}{ccccccc}
\hline Joint & $\theta$ & $\alpha$ & $a$ & $d$ & $\operatorname{var} q$ & Mass \\
\hline 1 & 0 & 0 & $q_{1}$ & 0 & $a$ & $31 \mathrm{~kg}$ \\
2 & 0 & 0 & $0.4 \mathrm{~m}$ & 0 & $\theta$ & $2 \mathrm{~kg}$ \\
3 & 0 & 0 & $0.2 \mathrm{~m}$ & 0 & $\theta$ & $2 \mathrm{~kg}$ \\
4 & 0 & 0 & $0.2 \mathrm{~m}$ & 0 & $\theta$ & $1 \mathrm{~kg}$ \\
\hline
\end{tabular}

Table 1. Denativ-Hartenberg parameters for planar experimental manipulator.

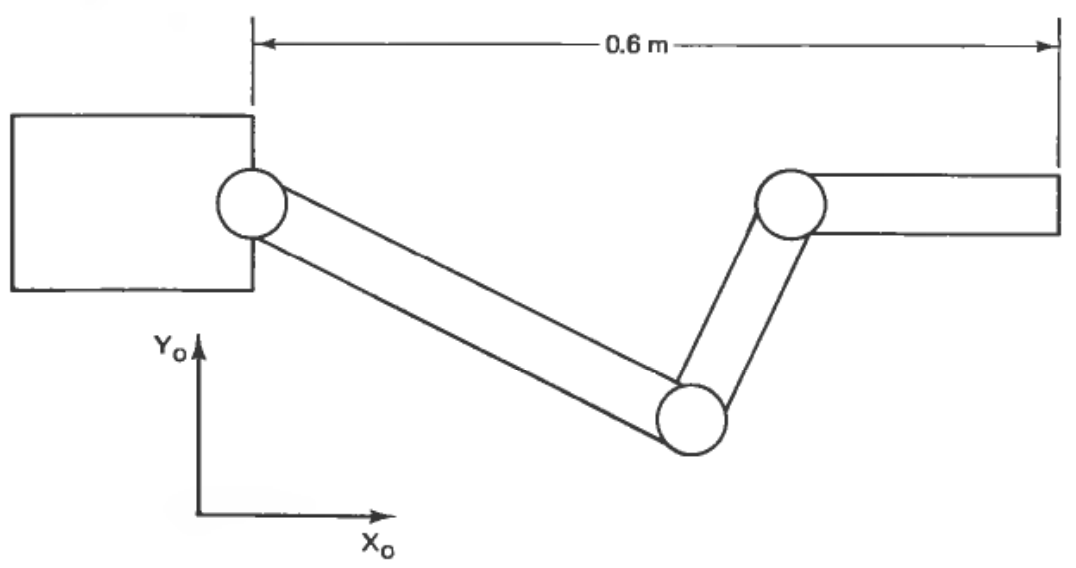

Figure 2. Initial position in the simulations.

orientate the end effector. The start position is shown in Fig. 2, from which a saw-tooth reference was given in $x$-direction, while the other end-effector references were held constant.

The redundancy was resolved so that the end-effector was placed in the middle of its workspace, which for this particular end-effector reference gives a macro part reference

$$
q_{1}=x_{e e}-0.6 \mathrm{~m} \text {. }
$$




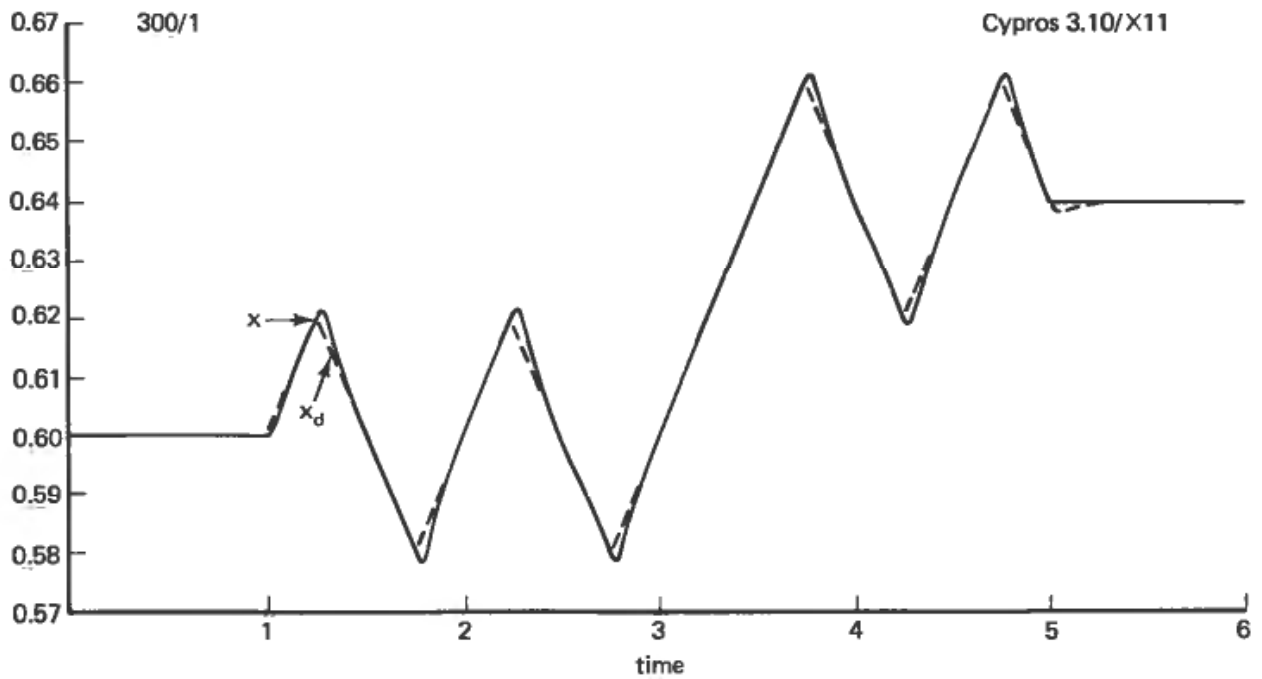

Figure 3. End-effector reference and position trajectory for planar manipulator.

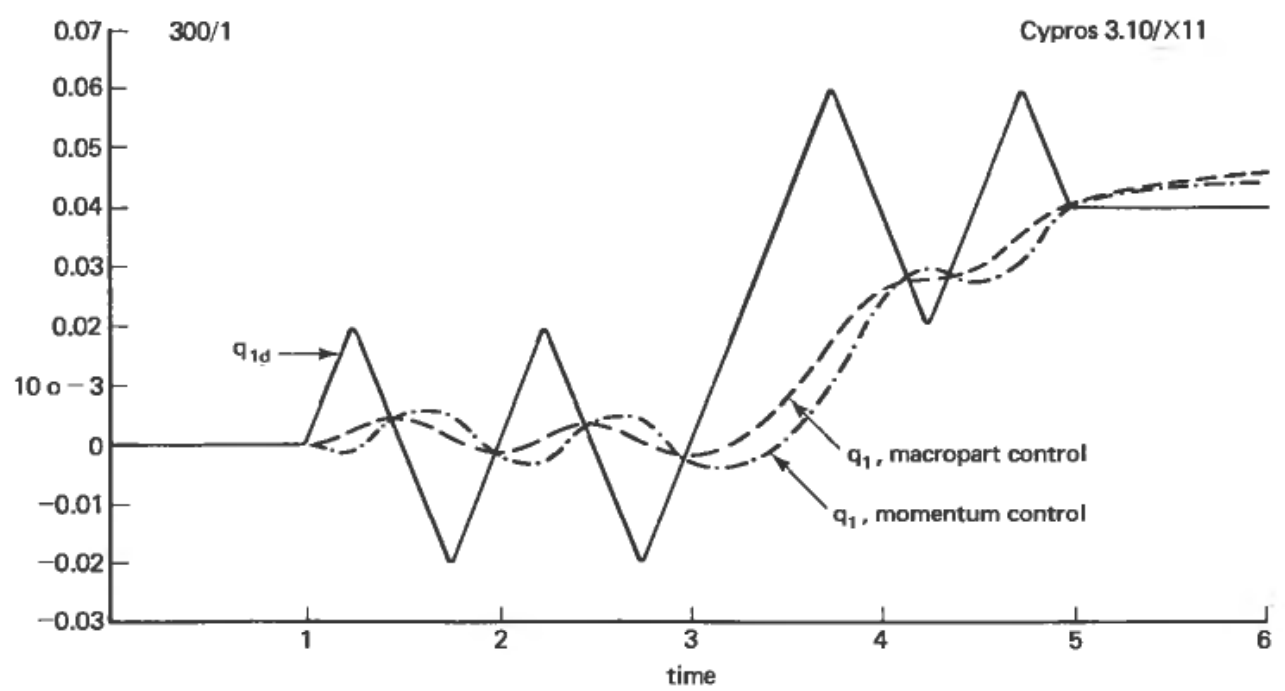

Figure 4. Macro part reference and position trajectories for planar manipulator with two different control augmentations.

From eqn. (10) the $x$-component of the linear momentum is the element which is most significant and was therefore a natural choice for the secondary task variable. The endeffector reference and position trajectories are shown in Fig. 3.

Since resolved acceleration control eqn. (6) is used, the end-effector tracking is the same whether we choose to control the position of the macro part directly or the centre of mass. The macro part, however, will have a slightly different behaviour, and this is demonstrated in Fig. 4. 
While the macro part motion does not differ so very much in the two cases, the difference in the control forces is significant. Figure 5 shows that although the endeffector tracking is the same, the peaks are much lower for the case with nonlinear decoupling of the linear momentum than for decoupling of macro part motion. This means that smaller motors may be used, still with the same accuracy. In Fig. 6, the time integrals of the two forces are shown. This is particularly interesting in satellite control, where the mass exerted by the reaction jets are proportional to the force, and less use of rocket fuel will prolong the spacecraft's working period.

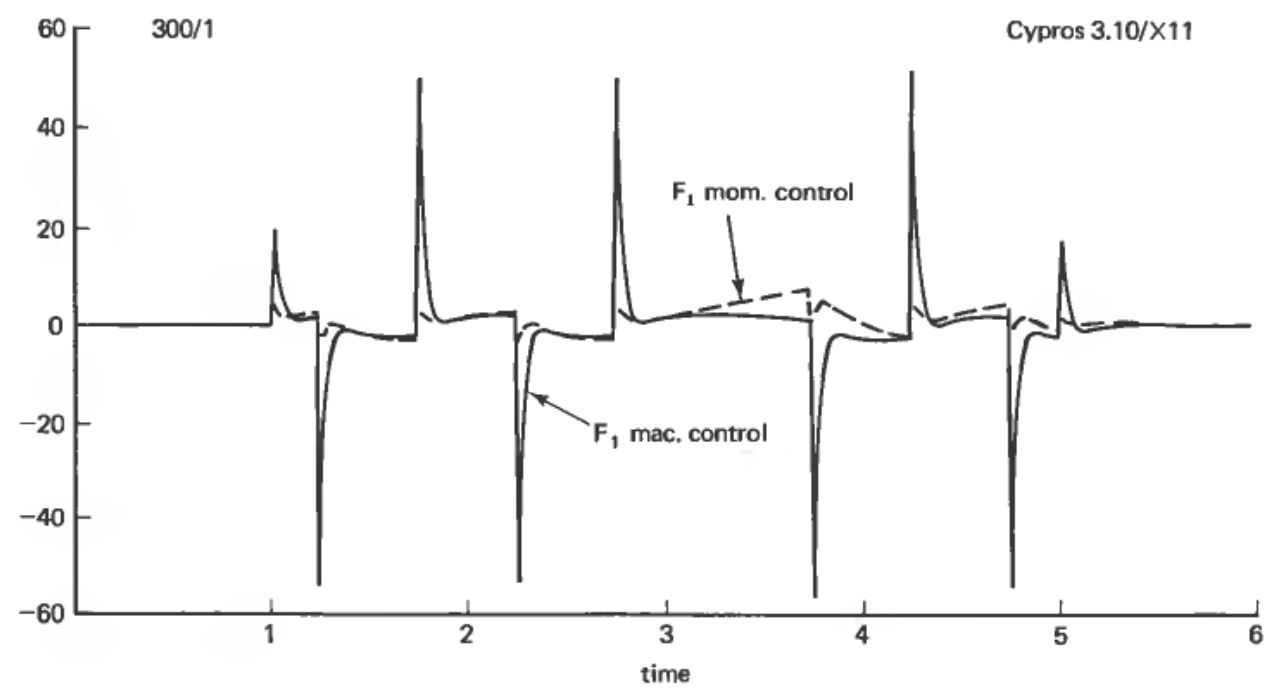

Figure 5. The forces on the macro part.

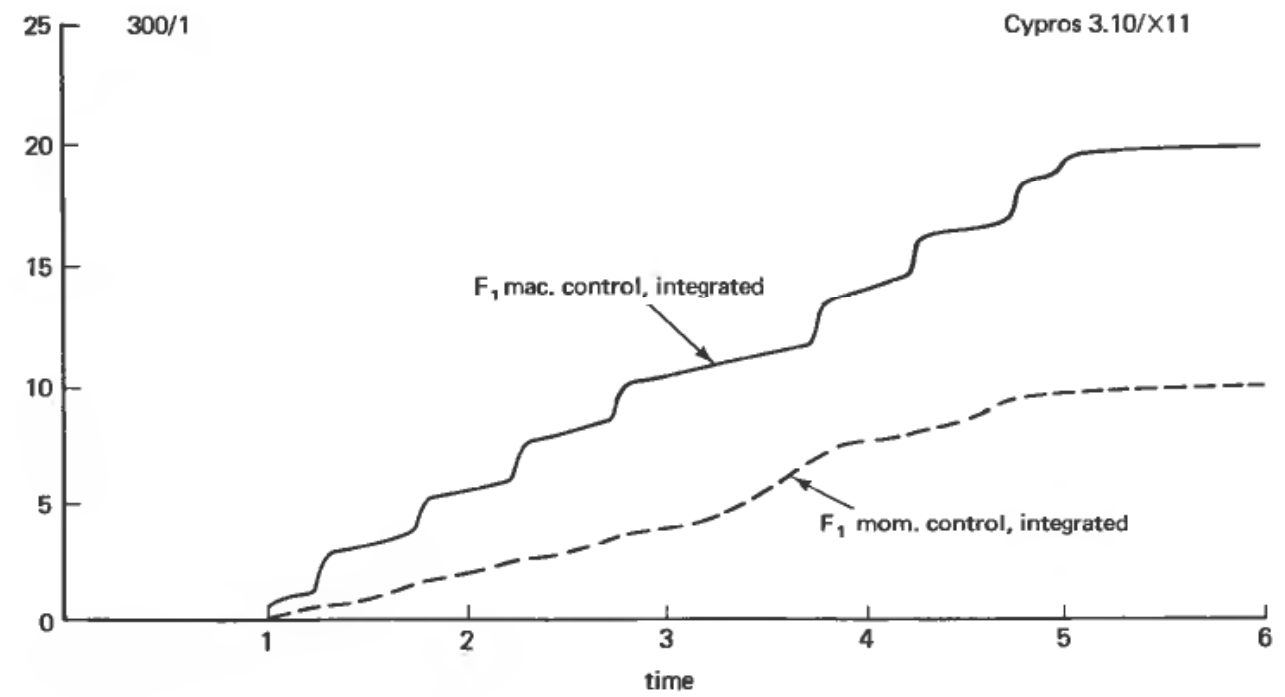

Figure 6. The time integral of the forces. 


\section{Spacecraft with manipulator}

A spacecraft-manipulator system is considered as a macro-micro manipulator system where both the macro- and the micro part have six degrees of freedom. The 6-dimensional vector $\dot{\boldsymbol{q}}_{M}$ from eqn. (1) is

$$
\dot{\boldsymbol{q}}_{M}=\left(\begin{array}{c}
{ }^{I} \dot{\boldsymbol{r}}_{0} \\
{ }^{I} \omega_{0}
\end{array}\right)
$$

where ${ }^{I} \dot{\boldsymbol{r}}_{0}$ is the position of the centre of mass and ${ }^{I} \omega_{0}$ is the angular velocity of the vehicle in inertial coordinates. The time integral of ${ }^{I} \omega_{0}$ has no physical meaning, so a $3 \times 3$ rotation matrix must be used to describe the orientation of the spacecraft. However, $q$ is used in the presentation to simplify the notation.

From eqn. (3) the 6-dimensional vector $\tau_{M}$ is the generalized forces from reaction wheels and thrusters in the spacecraft expressed in the $I$ frame, and must be transformed to the vehicle frame 0 where the forces are applied. $\tau_{\mu}$ is the 6-dimensional vector of manipulator torques. Equation (2) can be computed using the recursive Newton-Euler formulation (Luh, Walker and Paul (1980 b)) where the spacecraft is modeled as one joint with three translations and a second joint with three rotations.

The translational position of the end effector is given by ${ }^{I} \boldsymbol{r}_{6}$, while the orientation is given by the $3 \times 3$ rotation matrix ${ }_{6}^{I} R$ from frame 6 to frame $I$. For differential motion it is possible to define a 6-dimensional end-effector task vector by $\delta x_{e e}=\dot{x}_{e e} \delta t$ where $\delta t$ is a small time increment and

$$
\dot{\boldsymbol{x}}_{e e}=\left(\begin{array}{l}
I \dot{r}_{6} \\
I \omega_{6}
\end{array}\right)
$$

The $6 \times 12$ end-effector Jacobian matrix $J_{e e}$ can be calculated as proposed by Whitney (1972).

The motion of the spacecraft can be described in terms of the spacecraft position vector ${ }^{I} \boldsymbol{r}_{0}$ and the angular velocity ${ }^{I} \boldsymbol{\omega}_{0}$. This is used in the inverse kinematics. For the development of the dynamic controller it is, as for the planar manipulator, better to use the linear momentum ${ }^{\boldsymbol{I}} \boldsymbol{P}$. Here also the angular momentum ${ }^{\boldsymbol{I}} \boldsymbol{H}$ can be utilized.

\subsection{Control based on feedback linearization}

It is possible to achieve decoupling as in eqn. (6) for the 12 degree of freedom system by augmenting the 6-dimensional end-effector task vector $x_{e e}$ with a 6-dimensional task vector $x$, so that $x_{e e}$ and $x_{s}$ constitute the generalized coordinates of the spacecraftmanipulator system. It is then possible to define a $12 \times 12$ Jacobian which is nonsingular almost everywhere. Then, resolved acceleration control gives

$$
\begin{aligned}
\ddot{x}_{e e} & =u_{e e} \\
\ddot{x}_{s} & =u_{s}
\end{aligned}
$$

\subsection{Singularities}

In the general case, the augmented Jacobian $\boldsymbol{J}_{\boldsymbol{A}}$ may become singular in configurations where the end-effector has six degrees of freedom. For internal singularities, $\boldsymbol{J}_{\boldsymbol{A}}$ is singular when the manipulator is singular. The external singularities, however, will not be the same because of the reaction forces from manipulator to spacecraft. This is treated in Papadopoulos and Dubowsky (1989).

If a trajectory is commanded through a singularity it is possible to use a damped least-squares method (Maciejewski and Klein $(1985,1988)$ and Wampler $(1986))$ or a cancellation of the degenerate motion (Chiaverini and Egeland (1990)). 


\section{Simulation}

The performance of the proposed control strategy was investigated through simulations. A satellite with a manipulator similar to the PUMA type was simulated. The system had 12 degrees of freedom.

The satellite was modeled as a cube with $2 \mathrm{~m}$ long edges, and a homogeneously distributed mass of $200 \mathrm{~kg}$. The load was a point mass of $40 \mathrm{~kg}$ in the gripper. The Denavit-Hartenberg parameters for the manipulator and a sketch of the satellitemanipulator system are shown in Fig. 7. The manipulator inertia was simply point masses of $5 \mathrm{~kg}$ for every motor.

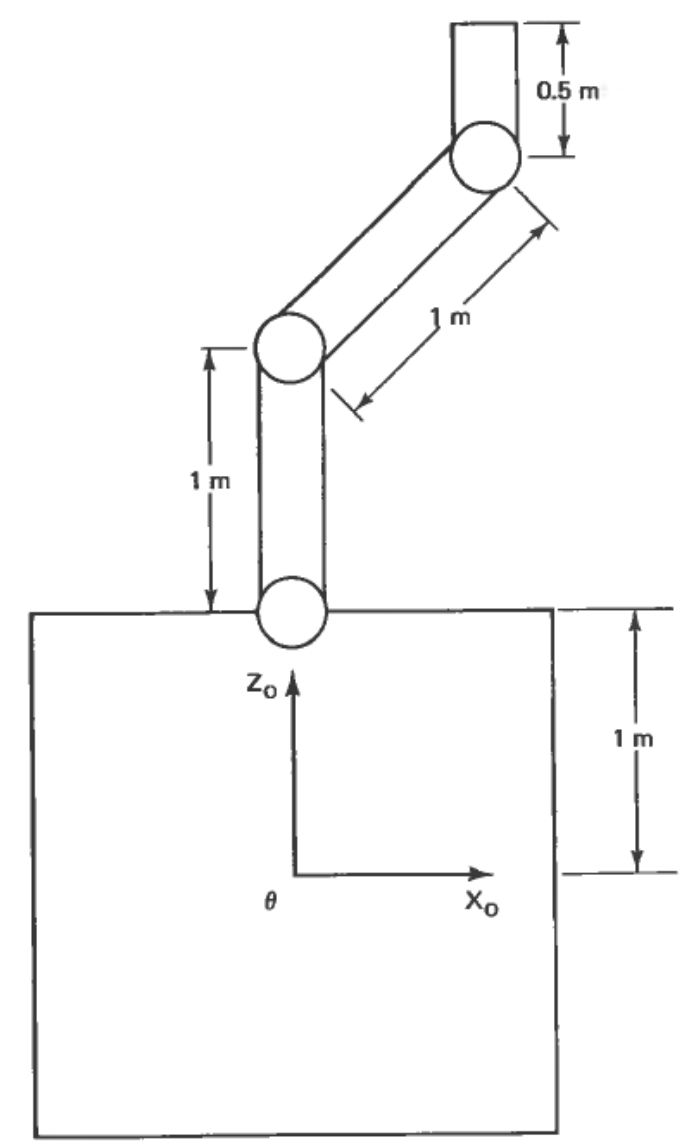

\begin{tabular}{crrcc}
\hline \multicolumn{5}{c}{ Denavit-Hartenberg parameters } \\
\hline Joint & $\theta$ & $\alpha$ & $a$ & $d$ \\
\hline 1 & 0 & 90 & 0 & 0 \\
2 & 90 & 0 & $1.0 \mathrm{~m}$ & 0 \\
3 & -90 & -90 & 0 & 0 \\
4 & 0 & 90 & 0 & $1.0 \mathrm{~m}$ \\
5 & 0 & -90 & 0 & 0 \\
6 & 0 & 0 & 0 & $0.5 \mathrm{~m}$
\end{tabular}

Figure 7. Satellite (side view) and manipulator system used in simulation. 

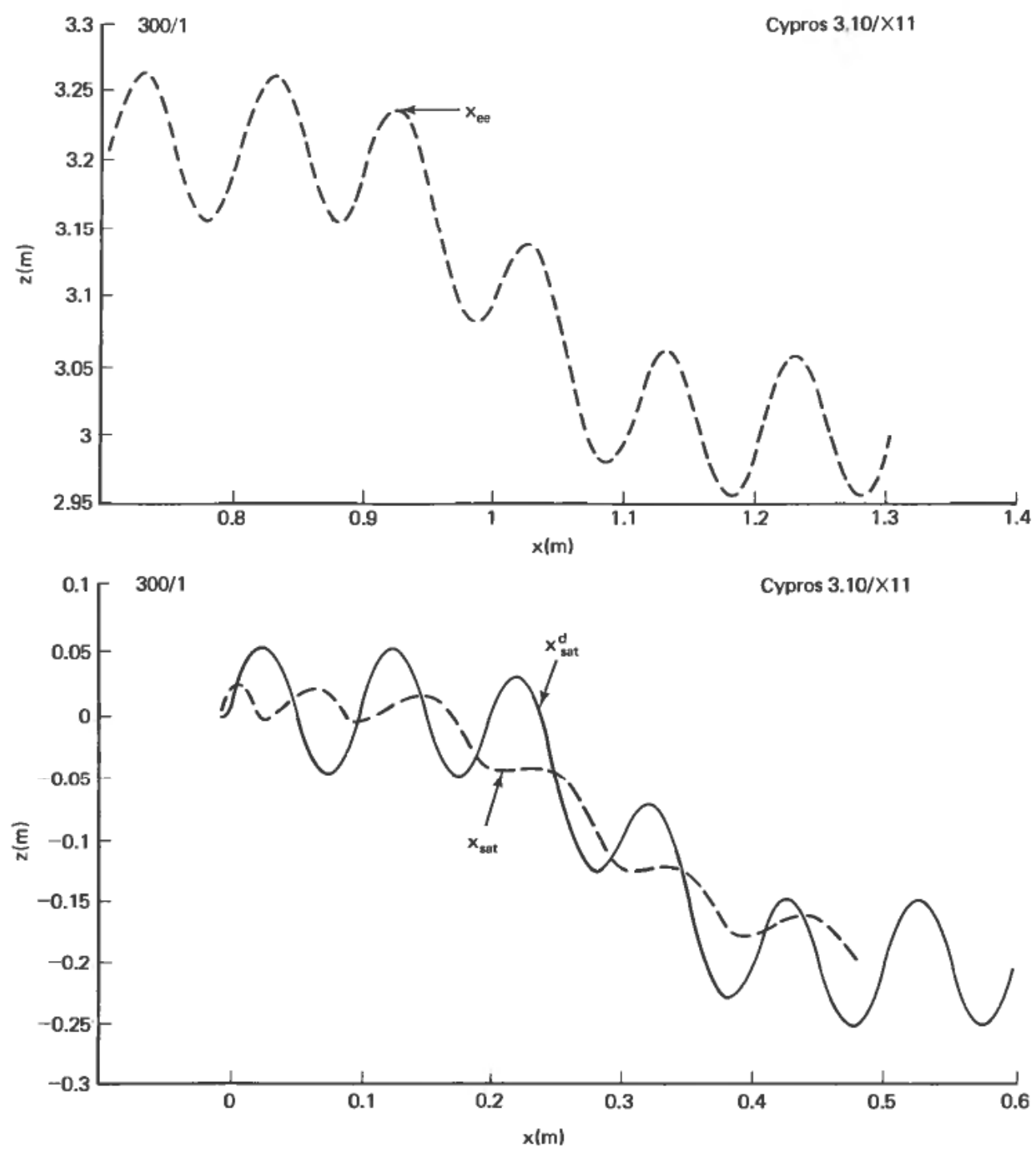

Figure 8. $x z$-plot of end-effector and satellite position when decoupling of $\dot{x}_{e e}$ and $\dot{\boldsymbol{x}}_{c m}$ is used. The end-effector tracks accurately, while the satellite motion is mainly caused by reaction forces from the manipulator. The slow gross positioning of the satellite is clearly demonstrated.

The end-effector reference was a stationary ramp in the $x$-direction,

$$
x_{d}=0 \cdot 7071+0 \cdot 1 t
$$

while in the $z$-direction the reference was a sum of a ramp and a small-amplitude sinusoidal component:

$$
z_{d}=\left\{\begin{aligned}
3 \cdot 2071+0.5 \sin (2 \pi t) & \text { if } 0<t<2 \\
3 \cdot 2071-0 \cdot 1(t-2)+0.05 \sin (2 \pi t) & \text { if } 2<t<4 \\
3.0071+0.05 \sin (2 \pi t) & \text { if } 4<t<6
\end{aligned}\right.
$$

The references in the remaining coordinates were constant. 
The redundancy resolution produced the reference for the satellite. The attitude reference for the satellite was equal to the orientation reference to the end-effector. The $y$-reference was also identical to the end-effector $y$-reference, while the $x$ and $z$ references for the satellite had constant offsets from the $x$ and $z$-references of the endeffector.

$$
\begin{aligned}
& x_{d}^{\text {sat }}=x_{d}-0.7071 \mathrm{~m} \\
& z_{d}^{\text {sat }}=z_{d}-3.2071 \mathrm{~m} .
\end{aligned}
$$

The system was simulated with $\boldsymbol{x}_{s}=\boldsymbol{x}_{c m}$ to achieve energy-efficient control. In this scheme, the end-effector is supposed to track accurately, while only a slow gross positioning is used for the satellite. Referring to eqns. (20) and (21) respectively, the end-effector loops had double poles at $-20 \mathrm{rad} / \mathrm{s}$, while the momentum loops had double poles at $-0.5 \mathrm{rad} / \mathrm{s}$.

The end-effector reference was tracked accurately, while fuel was not wasted in the satellite positioning by tracking the high frequency component of the trajectory. The results are shown in Fig. 8.

\section{Conclusion}

A control scheme for coordination of motion in macro-micro manipulator systems has been presented and tested in simulations. With decoupling of end-effector and total system momentums it was possible to achieve more efficient control, both less energy consuming and with smaller actuators. The end-effector tracked accurately, while a slow gross positioning was used for the macro part.

\section{ACKNOWLEDGEMENTS}

This work was supported by the Royal Norwegian Council for Scientific and Industrial Research through the Center for Robotics Research at the Norwegian Institute of Technology.

\section{REFERENCES}

Alexander, H. L. and Cannon, R. H. JR. (1987) Experiments on the control of a satellite manipulator. 1987 American Control Conf., Seattle, WA, June.

Chiaverini, S. and Egeland, O. (1990) A solution to the singularity problem in sixjoint manipulators. Proc. 1990 IEEE Int. Conf. Robotics and Automation, pp. 644-649.

DwYer, T. A. W., FADAL, M. S., ChEN, N. and LEe, G. F. K. (1988) Manipulator manoeuvering by feedback linearization with saturating inputs. Proc. 1985 IEEE Int. Conf. Robotics and Automation, pp. 947-953.

EGELAND, O. (1987) Task-space tracking with redundant manipulators. IEEE J. Robotics and Automation, 3, 471-475.

Egeland, O., SAGLl, J. R. and JANSEN, B. (1988) Optimal continuous-path control for manipulators with redundant degrees of freedom. Proc. IF AC Symp. Robot Control, Karlsruhe, West Germany, Oct. 1988.

Кнаттв, О. (1987) A unified approach for motion and force control of robot manipulators: The operational space approach. IEEE J. Robotics and Automation, 3, 43-53.

LONGMAN, R. W., LINDBERG, R. E. and ZeDD, M. F. (1987) Satellite-mounted robot manipulators-New kinematics and reaction moment compensation. Int. J. Robotics Research, 6, 87-103.

Luh, J. Y. S., Walker, M. W. and Paul, R. P. C. (1980a) On-line computational scheme for mechanical manipulators. ASME J. Dynamic Syst., Meas., Contr., 102, 69-76.

Luh, J. Y. S., WALKer, M. W. and PAul, R. P. C. (1980 b) Resolved acceleration control of mechanical manipulators. IEEE Trans. Automat. Contr., 25, 468-474. 
MaCiejewSKi, A. A. and KLeIN, C. A. (1985) Obstacle avoidance for kinematically redundant manipulators in varying environments. Int. J. Robotics Research, 4, 109-117.

MaCieJEwSKI, A. A. and KLeIN, C. A. (1988) Numerical filtering for the operation of robotic manipulators through kinematically singular configurations. J. Robotic Systems, 5, 527552.

NAKAMURA, Y. and HANAFUSA, H. (1986) Inverse kinematic solutions with singularity robustness for robot manipulator control. ASME J. Dynamic Syst., Meas., Contr., 108, 163-171.

Nakamura, Y., Hanafusa, H. and Yoshikawa, T. (1987) Task-priority based redundancy control of robot manipulators. Int. J. of Robotics Research, 6, 3-15.

NaKamurA, Y. and MuKHERJEe, R. (1989) Nonholonomic path planning of space robots. Proc. 1989 IEEE Int. Conf. Robotics and Automation, pp. 1050-1055.

PAPAdOPOUlos, E. and DUBOWSKy, S. (1989) On the dynamic singularities in the control of freefloating space manipulators. ASME Winter Annual Meeting, Dec. 1989, pp. 45-52.

Salisbury, J. K. and Abramowitz, J. D. (1988) Design and control of a redundant mechanism for small motion. Proc. 1985 IEEE Int. Conf. Robotics and Automation, pp. 323-328.

SPONG, M. W., THORP, J. S. and KLEINWAKS, J. M. (1986) The control of robot manipulators with bounded input. IEEE Trans. Automat. Contr., 31, 483-489.

VAFA, Z. and DUBOWSKY, S. (1987 a) On the dynamics of manipulators in space using the virtual manipulator approach. Proc. 1987 IEEE Int. Conf. Robotics and Automation, pp. 579-585.

VAFA, Z. and DUBOWSKY, S. (1987 b) Kinematic and dynamic models of manipulators in space: The concept of the virtual manipulator. Proc. 7th World Congress on the Theory of Machines and Mechanisms, Sevilla, Spain, Sept. 1987.

WAMPLER II, C. W. (1986) Manipulator inverse kinematic solutions based on vector formulations and dampled least-squares method. IEEE Trans. Syst., Man, Cybern., 16, 93-101.

WhitNeY, D. E. (1972) The mathematics of coordinated control if prosthetic arms and manipulators. ASME J. Dynamic Syst., Meas., Contr., 94, 303-309. 